\title{
A Miniature, One-Handed 3D Motion Controller
}

\author{
Kynan Eng \\ Institute of Neuroinformatics \\ University of Zurich and ETH Zurich \\ Winterthurerstrasse 190, CH-8057 Zurich, Switzerland \\ kynan@ini.phys.ethz.ch
}

\begin{abstract}
Users of three-dimensional computer-aided design (CAD) and gaming applications need to manipulate virtual objects in up to six degrees of rotational and translation freedom (DOF). To date, no 3D controller provides one-handed 6DOF input with miniature size and low cost. This paper presents a prototype of the first one-handed 6DOF motion controller suitable for use in portable platforms such as laptop computers, mobile telephones and hand-held game consoles. It is based on an optical sensor combined with novel planar spring mechanics, and can be easily manufactured using low-cost materials and processes.
\end{abstract}

Keywords: 3D motion controller, optical sensor, hand-held device.

\section{Introduction}

With the continued increase in the number of 3D computing applications in hand-held computers and mobile telephones, there is a need for miniature one-handed input devices combining small size with many input degrees of freedom (DOF). An ideal device would provide input in 6DOF ( 3 translation plus 3 rotation), be operable using only the thumb and index finger of one hand, be small enough to be mounted on a device such as a mobile telephone or ultra-portable laptop, provide reasonable accuracy and be cheap to manufacture.

There is currently no available device that satisfies all of the above requirements. Well-known input devices such as optical mice [1-3], trackballs [4] and mechanical or optical joysticks [5-7] output only 2 or 3 DOF. Multi-axis input devices based on accelerometers and digital compasses are also available, but accelerometers suffer from drift and digital compasses are expensive, sensitive to interference from external magnetic fields and cannot provide translation information. Full one-handed 6DOF input is available in systems using camera-based tracking of special patterns on an index object manipulated in free air [8-10], but they have problems with uncontrolled external lighting conditions, and are unsuitable for portable applications due to the unattached index object. A range of 6DOF input devices sold as the SpaceBall, SpacePilot and SpaceNavigator [11] solves these problems by using an arrangement of springs and optical sensors inside the device's hand grip, but the volume of the sensor does not allow it to be miniaturized for ultra-mobile applications.

\section{System Design}

To achieve practical one-handed input, a device must track operator-initiated movements of a manipulandum in multiple DOF and return it to a zero position when 
the operator lets go. Our device uses two key methods to achieve this functionality: visual tracking of index points on the manipulandum using standard low-cost imagers, and a specialized planar multiple spring arrangement for positioning the manipulandum. The arrangement of the components is shown in Figure 1.
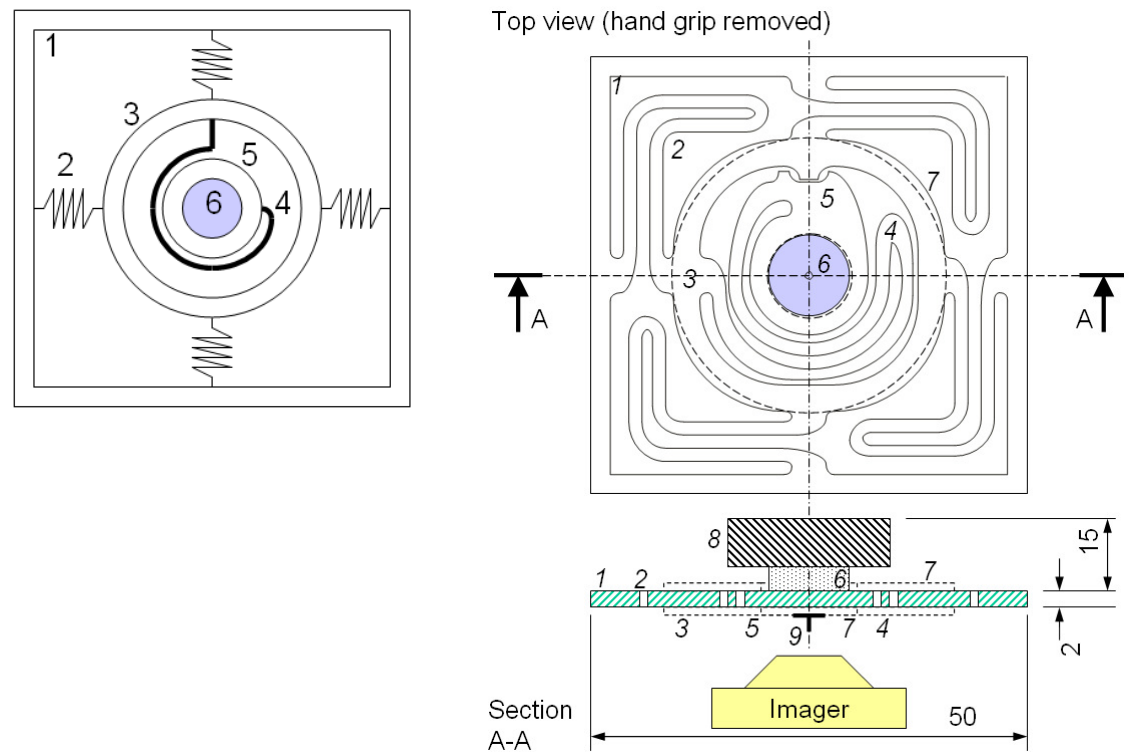

Fig. 1. (Left) Simplified view of the planar spring arrangement for the $3 \mathrm{D}$ controller. (Right) Overview of the design and layout of the 3D controller. The outer frame (1) holds the translational outer springs (2), which are connected to the inner frame (3) on which the torsional spring (4) is mounted. The center of the spring plate contains a mounting (5) for the grip shaft (6), which is located by means of restraining plates (7) attached to the inner frame above and below the spring plate. This arrangement allows the grip shaft to rotate but not translate relative to the inner frame, and prevents the torsional spring from bending out of the plane of the inner frame. The finger grip $(8,16-20 \mathrm{~mm}$ diameter) is rigidly attached to the grip shaft. The index points (9) are attached to the bottom of the grip shaft. All dimensions in $\mathrm{mm}$.

A standard low-cost USB webcam (Logitech, 320 x 240 pixels) facing the threedimensional array of index points performs image acquisition (Fig. 1 and Fig. 2). Standard image processing techniques are used detect the movements of the index points. To deduce the movements of the manipulandum, simple linear rules are used which consider the relative movements of the index points (Fig. 2). The index points are $0.5 \mathrm{~mm}$ in diameter and are spaced $2.0 \mathrm{~mm}$ apart. To eliminate zero-position error noise, which is a large problem for usability of all input devices, the software adapts the zero position of the index points at different rates depending on whether abovethreshold movement has been detected or not.

To improve user input precision while keeping size and manufacturing costs low, a planar multiple-spring arrangement was developed (Fig. 1). The multi-spring arrangement allows different restoring forces to be used for translation and rotation in and out of the plane, improving user ergonomics. In particular, it increases the out-of- 

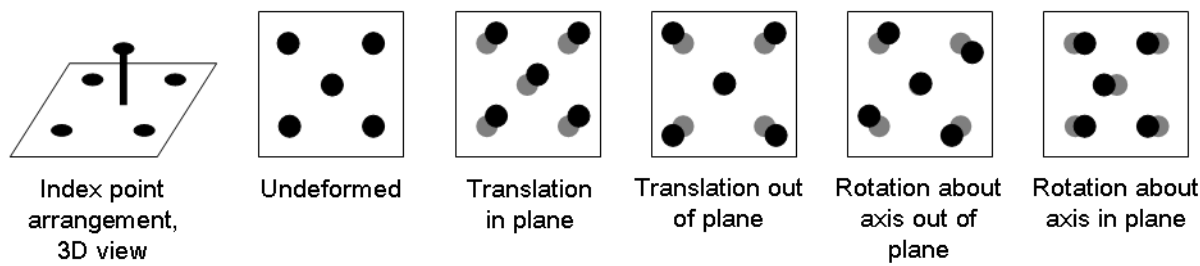

Rotation about axis in plane

Fig. 2. Arrangement of index points and 6DOF decoding of translations and rotations

plane stiffness of the system so that the force required to translate the manipulandum both in-plane and out-of-plane are within the same order of magnitude. The L-shaped outer springs ensure that in-plane diagonal and vertical/horizontal translation requires approximately the same amount of force. An enclosed torsional spring makes rotational movements easier, further improving usability.

\section{Prototype Testing}

A prototype of the device has been constructed and tested (Fig. 3). Laser-cut Plexiglas was used for the spring. The in-plane index points were printed on a laser printer. The out-of-plane index point was not used in this prototype, limiting the output of the system to 4DOF. Light-emitting diodes illuminated the index points.

The system's mechanical characteristics were measured: $2.2 \mathrm{~N} / \mathrm{mm}$ (horizontal plane $\mathrm{dX}, \mathrm{dY}$ ), $1.0 \mathrm{~N} / \mathrm{mm}$ (out of the plane $\mathrm{dZ}$ ) and $0.016 \mathrm{~N} / \mathrm{deg}$ (rotation about axis out of plane rotZ). Index point movements of $\sim 0.05 \mathrm{~mm}$ were reliably detected, corresponding to $\sim 4-5$ bit translation resolution and $\sim 3-4$ bits for rotation. This allowed users to position and orient a virtual cube (Fig. 2) in 4DOF with a little practice. The adaptation algorithm to eliminate zero position noise worked as designed. The PC CPU load (P4 2.8 GHz) was $20 \%$ including the graphics display.
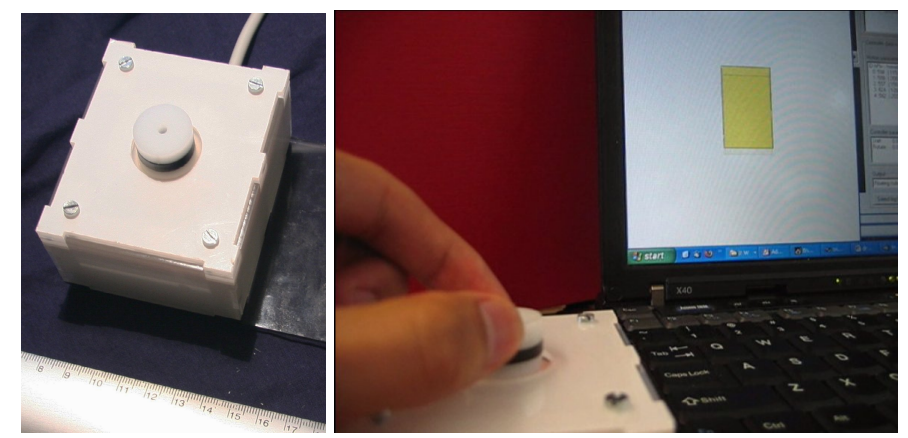

Fig. 3. Prototype controller (left) manipulating a virtual cube on a laptop screen (right)

\section{Conclusions and Outlook}

While the feasibility of the miniature 3D controller's working principles has been demonstrated, further work is required to achieve a truly usable mobile 6DOF device. 
A specialized low-profile webcam is needed to reduce the overall depth of the controller from $>40 \mathrm{~mm}$ to $<20 \mathrm{~mm}$. In addition, the out-of-plane calibration point needs to be added - its feasibility was verified in an earlier large-scale prototype, but so far it has proved difficult to reliably assemble a miniature version by hand. Further optimization of the spring design is also needed, in conjunction with a series of usability tests on a wide range of potential users of the device. Using higher resolution imagers (e.g. 1.3 megapixels) would improve sensitivity by about 2 bits, at the cost of increasing image processing requirements. Although the CPU demands of the image processing algorithms were low compared to the computing power available on current hardware, it may also be desirable to create an embedded version of the algorithm to create a stand-alone, platform-independent device

Acknowledgments. Thanks to Wolfgang Henggeler and Rodney Douglas for their helpful advice and encouragement with the construction of the system.

\section{References}

1. Jackson, S.B., Optical translator device, in US 4794384. 1988, Xerox Corporation (Stamford, CT, USA): USA.

2. Nestler, E. and T.F. Knight, Optical Mouse, in US 4799055. 1989, Symbolics Inc. (Cambridge, MA, USA): USA.

3. Adan, M.E., et al., Operator input device, in US 6,172,354, EP0572009A1, GB2272763, WO97/06506. 2001, Microsoft Corporation: US.

4. Jackson, S.B.: Optical translator device, in US 4794384, Xerox Corporation, Stamford, CT, USA (1988)

5. Nestler, E., Knight, T.F.: Optical Mouse, in US 4799055, Symbolics Inc., Cambridge, MA, USA (1989)

6. Adan, M.E., et al.: Operator input device, in US 6, 172, 354, EP057, A1, GB2272763, WO97/06506. 2001, Microsoft Corporation, US (2009)

7. Bidiville, M., et al.: Cursor pointing device utilizing a photodetector array with target ball having randomly distributed speckles, in United States Patent and Trademarks Office, United States of America (1994)

8. Liu, S.M.: Computer joy stick having two optical generating vector signals, in JP11259228A2, JP1003150, US6597453. Primax Electronics Ltd., JP (1999)

9. Shusuke, S.: Two-dimensional direction joy stick uni optical sensor. in JP06059737A2, priority JP1992000250358, Miura Takeshi, JP (1994)

10. Yoshimi, T.: Joy stick. In: JP61276014, priority JP19850118017., Fujitsu Ltd., JP (1986)

11. Arbter, K., Landzettel, K., Hirzinger, G.: Method and Input Device for Controlling the Position of an Object to be Graphically Displayed in Virtual Reality. In: US6545663, Deutsches Zeutrum fuer Luft- und Raumfahrt e. V., Koeln (DE), USA (2003)

12. Woods, E., Mason, P., Billinghurst, M.: MagicMouse: an Inexpensive 6-Degree-ofFreedom Mouse. In: Woods, E. (ed.) Proceedings of the 1st international conference on Computer graphics and interactive techniques in Australasia and South East Asia. 2003, ACM Press, Melbourne, AU (2003)

13. ARTookit (2007), cited; Available from: http://www.hitl.washington.edu/artoolkit/

14. 3DConnexion, Arrangement for the detection for relative movements or relative position of two objects: in USPTO: US2003/0103217 A1. 2003, 3DConnexion GmbH, Seefeld (DE) USA (2003) 